SINP-TNP/96-20

\title{
Density perturbation in extended inflation
}

\author{
S. Mallik ${ }^{a}$ and D. Rai Chaudhuri ${ }^{b}$ \\ a Saha Institute of Nuclear Physics, 1/AF, Bidhannagar, Calcutta 700064, India \\ ${ }^{b}$ Acharya Jagadish Chandra Bose College, 1/1B, AJC Bose Road, Calcutta 700020, India
}

\begin{abstract}
We examine the calculation of density perturbation at large scales in the inflationary scenario. The formula for its magnitude is reviewed from first principles and applied to the original model of extended inflation. Our estimate is an order of magnitude bigger than the earlier ones due to the difference in the time at which the primordial fluctuation is evaluated and to the inclusion of terms neglected earlier in the standard formula for density perturbation.
\end{abstract}

\section{Introduction}

One of the remarkable features of inflationary models [1] is that, besides solving the classical problems of large scale homogeneity and flatness, it also addresses the problem of creating the seeds of density inhomogeneity in the early universe. These are the quantum fluctuations in energy density during the inflationary epoch, which might have evolved to produce the observed density inhomogeneity in the universe. Much work has naturally been devoted to a study of the evolution of the initial density perturbation [2, 3, , [1].

The difficulty here is that of constructing a realistic model [5]. In the original, the 'old' inflationary model [6], the bubbles of true phase, nucleated during the strongly first order phase transition, cannot percolate. The slow-rollover models [7] require too small a coupling constant for reproducing the observed density fluctuation to cause problem of reheating. Finally the so-called 'chaotic' models [8] require initial conditions having no resemblance to the thermal equilibrium condition (or definite departures therefrom), usually believed to prevail in the early universe.

The extended inflationary models [9] are similar to the 'old' model with the Einstein gravity replaced by the theory of Jordan [10] and of Brans and Dicke [11]. The idea is to take advantage of the time dependence of gravitational 'constant' to solve the bubble nucleation problem. Though a realistic model still eludes construction [12], further work in this direction is expected to lead to such a model without violating astrophysical observations $[13$.

In this work we examine the evaluation of density perturbation in the inflationary epoch 14. We first relate the density perturbation to the quantum fluctuations in the energy density operator from first principles [15, 16]. We then consider the original model of extended 
inflation in the so-called Einstein frame [17]. By restricting the energy density operator to terms linear in the scalar field, the quantum fluctuations in the density reduce essentially to the two point function of the scalar field. The density perturbation turns out to be an order of magnitude bigger than the earlier evaluations [18, 19].

In sec. 2 we derive the formula for the density perturbation from first principles. In sec. 3 we consider the original model of extended inflation and derive the two point function for the scalar field. This is used to evaluate the density perturbation in sec. 4 . In sec. 5 we identify the points of difference between the present and the earlier evaluations leading to an order of magnitude difference. Finally in sec. 6 we summarise our work emphasising these differences.

\section{Density fluctuation formula}

We begin by reviewing the definitions of density inhomogeneity and quantum fluctuation to relate them from first principles [15, 16]. Let $\rho(\vec{x}, t)$ be the energy density field of the Universe, which we assume for simplicity to be confined within a (large) volume $V$. The mean square fluctuation in $\rho(\vec{x}, t)$ is defined as

$$
\left(\frac{\delta \rho}{\rho}\right)^{2}=\left\langle\left(\frac{\rho(\vec{x}, t)-\bar{\rho}(t)}{\bar{\rho}(t)}\right)^{2}\right\rangle_{\vec{x}},
$$

where $\langle\cdots\rangle_{x}$ denotes average over all space and $\bar{\rho}(t)=\langle\rho(\vec{x}, t)\rangle_{x}$, the averaged, homogeneous background density. One writes

$$
\rho(\vec{x}, t)=\bar{\rho}(t)(1+\delta(\vec{x}, t))
$$

where the density contrast $\delta(\vec{x}, t)$ has the Fourier expansion

$$
\delta(\vec{x}, t)=\frac{1}{\sqrt{V}} \sum_{k} \delta_{k}(t) e^{-i \vec{k} \cdot \vec{x}} .
$$

Then the mean square fluctuation (2.1) becomes

$$
\left(\frac{\delta \rho}{\rho}\right)^{2}=\frac{1}{V} \sum_{k}\left|\delta_{k}(t)\right|^{2} \rightarrow \int \frac{k^{3}\left|\delta_{k}(t)\right|^{2}}{2 \pi^{2}} d(\ln k) .
$$

The so-called fluctuation power per logarithmic interval in wavenumber is defined as

$$
\left(\frac{\delta \rho}{\rho}\right)_{k}^{2}=\frac{k^{3}\left|\delta_{k}(t)\right|^{2}}{2 \pi^{2}} .
$$

For our purpose it is useful to consider a related quantity, namely, the mean square mass fluctuation on a given length scale. It measures the mass fluctuation within a certain volume $v$ by averaging the squared excess mass in it over all points $x_{0}$ throughout the volume $V$ of the universe. To avoid sharp boundary, one smears $v$ with a Gaussian window function. The mass within such a smeared sphere placed at $\vec{x}_{0}$ is

$$
m_{l}\left(\vec{x}_{0}, t\right)=\int d^{3} y e^{-y^{2} / 2 l^{2}} \rho\left(\vec{x}_{0}+\vec{y}, t\right)
$$


The mean square mass fluctuation on length scale $l$ is then

$$
\left(\frac{\delta m}{m}\right)_{l, c}^{2}=\left\langle\left(\frac{m_{l}\left(\vec{x}_{0}, t\right)-\bar{m}_{l}(t)}{\bar{m}_{l}(t)}\right)^{2}\right\rangle_{\vec{x}_{0}} .
$$

Here $\bar{m}_{l}(t)=<m_{l}\left(\overrightarrow{x_{0}}, t\right)>_{\overrightarrow{x_{0}}}$, the average mass obtained by replacing $\rho$ by $\bar{\rho}$ in (2.6). The subscript c stands for classical and indicates the phenomenological nature of the evaluation. Inserting (2.3) in (2.6) we get

$$
\left(\frac{\delta m}{m}\right)_{l, c}^{2}=\int \frac{d^{3} k}{(2 \pi)^{3}}\left|\delta_{k}(t)\right|^{2} e^{-k^{2} l^{2}}
$$

Now suppose that this density inhomogeneity has a quantum origin in the inflationary epoch. Then it should be calculable by considering the quantum fluctuations in the energy density operator, $T_{00}$. This operator will be written explicitly for the specific Lagrangian of extended inflation considered in the next section. Here we write it as a sum of two parts,

$$
T_{00}(\vec{x}, t)=\bar{\rho}(t)+U(\vec{x}, t),
$$

where $\bar{\rho}$, the classical part, is to be identified with the background density considered above and $U$, the quantum part, depends on the quantum field operator.

In analogy with (2.6) let us define the mass operator,

$$
\hat{m}_{l}(t)=\int d^{3} x e^{-x^{2} / 2 l^{2}} T_{00}(\vec{x}, t),
$$

where the hat on $m$ emphasises that it is an operator. The mean squared fluctuation in mass in the smeared sphere is

$$
\left(\frac{\delta m}{m}\right)_{l, q}^{2}=\left\langle\left(\frac{\hat{m}_{l}(t)-\bar{m}_{l}(t)}{\bar{m}_{l}(t)}\right)^{2}\right\rangle,
$$

where $\langle\cdots\rangle$ denotes the quantum mechanical expectation value in the state with energy density $\bar{\rho}(t)$, which corresponds to the vacuum of the conventional quantum field theory. The subscript $q$ distinguishes it from the phenomenological evaluation in (2.7-8). Inserting the Fourier transform of the two point function of $U$

$$
\left\langle U(\vec{x}, t) U\left(\overrightarrow{x^{\prime}}, t\right)\right\rangle=\int \frac{d^{3} k}{(2 \pi)^{3}} e^{-i \vec{k} \cdot\left(\vec{x}-\overrightarrow{x^{\prime}}\right)} D_{k}(t),
$$

in eqn (2.11), we get

$$
\left(\frac{\delta m}{m}\right)_{l, q}^{2}=\int \frac{d^{3} k}{(2 \pi)^{3}} e^{-k^{2} l^{2}} \frac{D_{k}(t)}{\bar{\rho}^{2}(t)} .
$$

If we now identify the spatial averaging in (2.7) with the quantum mechanical expectation value in (2.11), we get the desired relation,

$$
\left|\delta_{k}(t)\right|^{2}=\frac{D_{k}(t)}{\bar{\rho}^{2}(t)}
$$


allowing us to calculate the density fluctuation during inflation, when it is still within the Hubble length (causal horizon). Its evolution as superhorizon-sized perturbation, from the time $t_{h}$ when it leaves the Hubble radius until its re-entry within it later, is described by the constancy of the quantity $\zeta=\delta_{k}(t) /(1+\bar{p} / \bar{\rho})$ [ [4]. Assuming radiation dominance at the time of re-entry, we finally get the density perturbation at that time as

$$
\left(\frac{\delta \rho}{\rho}\right)_{H}=\frac{4 \sqrt{k^{3} D_{k}\left(t_{h}\right)}}{3 \sqrt{2} \pi(\bar{\rho}+\bar{p})_{t_{h}}}
$$

\section{Extended inflation in Einstein frame}

Because of the nonminimal coupling of the scalar field in the original Brans-Dicke (BD) Action [1], it is not possible to do calculations with it in the canonical framework of quantum field theory. It has, however, been shown [17] that an appropriate Weyl rescaling [20] can transform this action to a form where both the gravity and the kinetic term in the scaled BD field are canonical. In this so-called Einstein frame, the rescaled action becomes

$$
S=\int d^{4} x \sqrt{g}\left(\frac{R}{16 \pi G}+\frac{1}{2} g^{\mu \nu} \partial_{\mu} \Psi \partial_{\nu} \Psi-V(\Psi)\right)
$$

where $\Psi(x)$ is the BD field in the Einstein frame and

$$
V(\Psi)=M^{4} e^{-2 \Psi / \psi_{0}}, \quad \psi_{0}=\sqrt{\frac{2 \omega+3}{16 \pi}} m_{P},
$$

$m_{P} \equiv G^{-1 / 2}$ being the present value of the Planck mass. This form for the potential function results from assuming the matter Lagrangian in the original (Jordan) frame to be dominated by the false vacuum energy density, $M^{4}$. The dimensionless parameter $\omega$ appears in the original BD Lagrangian.

We decompose the BD field $\Psi$ into a homogeneous, classical field $\psi$ and a quantum field $\phi$

$$
\Psi(\vec{x}, t)=\psi(t)+\phi(\vec{x}, t)
$$

In the spatially flat FRW metric, $d s^{2}=d t^{2}-a(t)^{2} \overrightarrow{d x}^{2}$, the classical equations of motion for $\psi(t)$ and the scale factor $a(t)$ are

$$
\begin{gathered}
\ddot{\psi}+3 \frac{\dot{a}}{a}+\frac{d V}{d \psi}=0 \\
\left(\frac{\dot{a}}{a}\right)^{2}=\frac{8 \pi}{3 m_{P}^{2}}\left(\frac{1}{2} \dot{\psi}^{2}+V(\psi)\right)
\end{gathered}
$$

In the Einstein frame, the extended inflation resembles a slow-rollover inflation off an exponential potential 18].

The solution to the coupled equations are [9, 17]

$$
\begin{gathered}
\psi(t)=\psi(0)+\psi_{0} \ln (1+C t), \\
a(t)=a(0)(1+C t)^{p}, \quad p=(2 \omega+3) / 4 .
\end{gathered}
$$


where

$$
C=\frac{2 M^{2}}{q \omega m_{P}} e^{-\psi(0) / \psi_{0}}, \quad q \omega=\sqrt{\frac{(6 \omega+5)(2 \omega+3)}{32 \pi}} .
$$

We note that the Hubble length in this model is,

$$
H(t)^{-1}=\left(\frac{\dot{a}}{a}\right)^{-1}=\frac{1+C t}{C p} .
$$

We now turn to the quantum theory. Expanding the Lagrangian around the classical field $\psi$, one gets an (infinite) series in powers of the quantum field $\phi$, though the coefficient of expansion $\left(M / m_{P}\right)$ is small. Such a Lagrangian is, of course, not perturbatively renormalisable. Here we simply reject all higher order terms retaining, in fact, only the lowest (second) order terms to get

$$
S_{q}=\int d^{3} x d t a^{3}(t)\left(\frac{1}{2} \dot{\phi}^{2}-\frac{1}{2 a^{2}}(\nabla \phi)^{2}-\frac{1}{2} \mu^{2}(t)\right)
$$

where

$$
\mu^{2}(t)=\frac{d^{2} V}{d \psi^{2}}=\frac{2(3 p-1)}{p^{2}} H^{2}(t)
$$

We calculate the Feynman propagator for $\phi$ in the classical background field $\psi(t)$. Its Fourier transform in 3-space,

$$
\left\langle T \phi(\vec{x}, t) \phi\left(\overrightarrow{x^{\prime}}, t^{\prime}\right)\right\rangle=\int \frac{d^{3} k}{(2 \pi)^{3}} e^{-i \vec{k} \cdot\left(\vec{x}-\overrightarrow{x^{\prime}}\right)} G_{k}\left(t, t^{\prime}\right),
$$

satisfies the inhomogeneous equation

$$
\left(\frac{d^{2}}{d t^{2}}+3 \frac{\dot{a}}{a} \frac{d}{d t}+\frac{k^{2}}{a^{2}}+\mu^{2}(t)\right) G_{k}\left(t, t^{\prime}\right)=-\frac{i}{a^{3}(t)} \delta\left(t-t^{\prime}\right) .
$$

The Green's function can be constructed by the familiar procedure. Let $f^{(+)}(t), f^{(-)}(t)$ be two linearly independent mode functions satisfying the homogeneous part of the above equation and incorporating the boundary conditions that $f^{( \pm)}$contain respectively positive and negative frequencies during the initial period for not too low physical momenta [21]. Their normalisation is fixed by the value of the Wronskian of the two solutions derivable from (3.10). Then we have

$$
G_{k}\left(t, t^{\prime}\right)=f^{(+)}(t) f^{(-)}\left(t^{\prime}\right) \theta\left(t-t^{\prime}\right)+f^{(-)}(t) f^{(+)}\left(t^{\prime}\right) \theta\left(t^{\prime}-t\right) .
$$

The equation for the mode functions can be solved exactly. With a change of variable [22], $\tau=(1+C t)^{1-p} /(p-1)$, it becomes

$$
\left(\frac{d^{2}}{d \tau^{2}}-\frac{2 p}{p-1} \frac{1}{\tau} \frac{d}{d \tau}+\kappa^{2}+\frac{2(3 p-1)}{(p-1)^{2} \tau^{2}}\right) f^{( \pm)}(\tau)=0, \quad \kappa=\frac{k}{a(o) C} .
$$

It is now easy to cast it in the standard form of Bessel's equation. We get

$$
f^{(+,-)}(t)=\frac{1}{2} \sqrt{\frac{p}{p-1} \frac{\pi}{H}} \frac{H_{\nu}^{(1,2)}(z)}{a(t)^{3 / 2}},
$$


where $H_{\nu}^{(1,2)}(z)$ are the Hankel functions of the first and second kind respectively [23, 24] and

$$
\nu=\frac{\sqrt{3(3 p-1)(p-3)}}{2(p-1)}, \quad z=\kappa \tau=\frac{p}{p-1} \frac{k}{a H} .
$$

Two point functions involving $\phi$ and $\dot{\phi}$ are immediately obtainable from (3.11) and (3.13).

We also write here the expressions for the energy density and pressure, which can be obtained directly from the energy-momentum tensor,

$$
T_{\mu \nu}=\partial_{\mu} \Psi \partial_{\nu} \Psi-g_{\mu \nu}\left(\frac{1}{2} g^{\alpha \beta} \partial_{\alpha} \Psi \partial_{\beta} \Psi-V(\Psi)\right),
$$

for the action (3.1). We have already split the energy density as the sum of a homogeneous, classical part and a quantum part in (2.9). Inserting (3.2) in (3.14), they are

$$
\begin{gathered}
\bar{\rho}=\frac{1}{2} \dot{\psi}^{2}+M^{4} e^{-2 \psi(0) / \psi_{0}}=\frac{3}{8 \pi} m_{p}^{2} H^{2}, \\
U=-\frac{M^{4}}{\psi_{0}^{2}} \phi+\dot{\psi} \dot{\phi} \\
=-\frac{m_{p} H^{2}}{\sqrt{\pi(2 \omega+3)^{3}}}\left[(6 \omega+5) \phi+(2 \omega-1) \tau \frac{d}{d \tau} \phi\right] .
\end{gathered}
$$

In $U$ we have only retained terms linear in $\phi$. Higher order terms like $\phi^{2}$ would give rise to loops and hence divergence in the expression for the density fluctuation. As already stated, such terms are multiplied by small coefficients. Thus in any reasonable renormalisation scheme , such terms are expected to produce small contributions. We also note the expression for the homogeneous pressure,

$$
\bar{p}=\frac{1}{2} \dot{\psi}^{2}-M^{4} e^{-2 \psi(0) / \psi_{0}}=-\frac{1}{8 \pi} \frac{6 \omega+1}{2 \omega+3} m_{P}^{2} H^{2} .
$$

\section{Evaluation of $\delta \rho / \rho$}

Since the energy density operator $U(\phi)$ is linear in $\phi$, it is simple to express the two point function of $U(\phi)$ in terms of the mode functions $f^{( \pm)}(t)$. Noting the symmetry of Hankel functions, $H_{\nu}^{(2)}(z)=H_{\nu}^{(1) *}(z)$ for real $z$, we can write its Fourier transform (2.12) as

$$
\begin{aligned}
D_{k}(t) & =\frac{m_{p}^{2} H^{4}}{\pi(2 \omega+3)^{3}}\left|(6 \omega+5) f^{(+)}(\tau)+(2 \omega-1) \tau \frac{d}{d \tau} f^{(+)}\right|^{2}, \\
& =\frac{m_{p}^{2}}{4(2 \omega-1)(2 \omega+3)^{2}}\left(\frac{H(t)}{a(t)}\right)^{3}|F(t)|^{2},
\end{aligned}
$$

where

$$
F(t)=\frac{3}{2}(6 \omega+5) H_{\nu}^{(1)}(z)+(2 \omega-1) z \frac{d}{d z} H_{\nu}^{(1)}(z) .
$$


Let $\lambda_{\text {phys }}(t)$ be the physical wavelength characterising the density perturbation at time $t$, belonging to the comoving wavenumber $k, \lambda_{\text {phys }}(t)=2 \pi a(t) / k$. At time $t_{h}$ during inflation, when it equals the Hubble length, its magnitude is $\lambda_{\text {phys }}\left(t_{h}\right)=H^{-1}\left(t_{h}\right)$, so that $k /\left.(a H)\right|_{t_{h}}=$ $2 \pi$. Then eq.(2.15) becomes

$$
\left(\frac{\delta \rho}{\rho}\right)_{H}=\frac{4 \pi^{3 / 2}}{3 \sqrt{2 \omega-1}} \frac{H\left(t_{h}\right)}{m_{p}}\left|F\left(t_{h}\right)\right| .
$$

At time $t_{h}$ the argument of the Hankel function becomes $z\left(t_{h}\right)=2 \pi p /(p-1)$, a value large enough to justify the use of asymptotic expansion [23]. Retaining terms in $z$ upto the first nonleading one, it is simple to evaluate $F\left(t_{h}\right)$ giving

$$
F\left(t_{h}\right)=2 \sqrt{(2 \omega-1)(2 \omega+3)} K(\omega),
$$

where $K$ is of order unity,

$$
K^{2}(\omega)=1+\frac{3\left(20 \omega^{2}+48 \omega+27\right)}{4 \pi^{2}(2 \omega+3)^{2}} .
$$

To determine $t_{h}$, we follow earlier authors [18, 19] to make certain simplifying assumptions. It is assumed that the extended inflation ends at time $t_{e}$ and instantly gives rise to the radiation dominated era with an initial temprerature $T \simeq M$. Further, since the gravitational 'constant' ceases to vary in the radiation era, the (classical) BD field $\psi$ is set equal to zero from the time $t_{e}$ onwards 25]. We then get from (3.5),

$$
\frac{M}{H\left(t_{e}\right)}=\frac{q \omega}{2 p} \frac{m_{p}}{M}
$$

The corresponding wavelengths at time $t_{h}$ and at the present time $t_{p}$ can be related as

$$
\lambda_{\text {phys }}\left(t_{h}\right)=\frac{a\left(t_{h}\right)}{a\left(t_{e}\right)} \frac{a\left(t_{e}\right)}{a\left(t_{p}\right)} \lambda_{\text {phys }}\left(t_{p}\right) .
$$

The scale factors can now be evaluated to give

$$
\frac{M}{H\left(t_{h}\right)}=\left(\frac{M / H\left(t_{h}\right)}{M / H\left(t_{e}\right)}\right)^{p} T_{p} \lambda_{p h y s}\left(t_{p}\right),
$$

where $T_{p}=2.7 \mathrm{~K}$, the present temperature of the background radiation. Solving for $M\left(t_{h}\right)$ and using (4.4) we get

$$
H\left(t_{h}\right)=M\left(\frac{2 p}{q \omega} \frac{M}{m_{P}}\right)^{\frac{p}{(p-1)}}\left(T_{p} \lambda_{p h y s}\left(t_{p}\right)\right)^{\frac{1}{(p-1)}} .
$$

Inserting this value in (4.2) we finally get

$$
\left(\frac{\delta \rho}{\rho}\right)_{H}=\frac{8 \pi}{3} K \sqrt{2 \pi}[(2 \omega+3) / 2]^{\frac{6 \omega+5}{2(2 \omega-1)}}(q \omega)^{-\frac{2 \omega+3}{2 \omega-1}}\left(M / m_{P}\right)^{\frac{2(2 \omega+1)}{2 \omega-1}}\left(T_{p} \lambda\left(t_{p}\right)\right)^{\frac{4}{2 \omega-1}} .
$$

This result differs from what one finds in the literature [18, 19]. In particular it is larger than the result by Guth and Jain [19] by a factor of $8 \pi K / 3 \simeq 10$. The sources of this difference are discussed in the next section. 


\section{Comparison with earlier works}

There are two major differences with earlier works, which contribute to the enhancement of our result. One is the time at which the mode functions are evaluated. As already stated, the argument of the Hankel functions around the time of Hubble length crossing is sufficiently large for the asymptotic expansion to apply,

$$
H_{\nu}^{1,2}(z) \rightarrow \sqrt{\frac{2}{\pi z}} e^{ \pm i z}, \quad(z \operatorname{large}) .
$$

Then the fluctuation in these oscillatory Fourier components of the quantum field is given by

$$
\frac{k^{3}\left|f^{ \pm}(t)\right|^{2}}{2 \pi^{2}}=\left(\frac{k}{a H} \frac{H}{2 \pi}\right)^{2}
$$

which reduces to just $H^{2}$ at the time of horizon exit, independently of the value of $\nu$.

But in the literature, the evaluation at the Hubble length crossing is actually carried out when the wavelength of the perturbation is much larger than the Hubble length and the corresponding mode ceases to oscillate. In this 'frozen' state, the argument of the Hankel functions is small enough for expansion around the origin to apply,

$$
H_{\nu}^{(1,2)}(z) \rightarrow \pm i \frac{\Gamma(\nu)}{\pi}\left(\frac{z}{2}\right)^{-\nu}, \quad(z \text { small })
$$

Further $\nu$ is assumed very close to $\frac{3}{2}$. Under these conditions the fluctuation in the quantum field is given by

$$
\left(\frac{k^{3}\left|f^{( \pm)}(t)\right|^{2}}{2 \pi^{2}}\right)^{(o)}=\left(\frac{p-1}{p} \frac{H}{2 \pi}\right)^{2} .
$$

The superscript $(o)$ indicates evaluation following literature. Thus our expression (5.2), though not of this form in general, does reduce to this form at the time of Hubble length crossing but is larger by a factor of $(2 \pi p /(p-1))^{2}$.

The other difference is the omission of the $\dot{\phi}$ term in the energy density operator. Thus in the literature the energy density fluctuation is related to the field fluctuation by

$$
\left(\frac{k^{3} D_{k}(t)}{2 \pi^{2}}\right)^{(o)}=\left(\frac{\partial V}{\partial \psi}\right)^{2}\left(\frac{k^{3}\left|f^{( \pm)}(t)\right|^{2}}{2 \pi^{2}}\right)^{(o)}
$$

Comparing with (4.1) we see that the neglected term is of the same order of magnitude as the one retained for $z=2 \pi p /(p-1)$.

At this point it is simple to obtain the standard expression for the density fluctuation and verify the enhancement factor. For the slow-rollover scenario, $(\partial V / \partial \psi)$ may be estimated from the classical equation of motion (3.3) as $(\partial V / \partial \psi)=-3 H \dot{\psi}$. Then inserting (5.4-5) into (2.15) we recover the standard formula,

$$
\left(\frac{\delta \rho}{\rho}\right)_{H}^{(o)}=\left(\frac{H^{2}}{\dot{\psi}}\right)_{t_{h}}
$$


on ignoring a factor of $2(p-1) / \pi p$. It can be immediately evaluated to give

$$
\left(\frac{\delta \rho}{\rho}\right)_{H}^{(o)}=\sqrt{\pi(2 \omega+3)} \frac{H}{m_{P}}
$$

This standard result is clearly smaller than our result (4.2-3) by the factor $8 \pi K / 3$.

\section{Discussion}

We review from first principles the formula for the density perturbation due to quantum fluctuations in the energy operator. We then evaluate the resulting formula in the context of the original model of inflation in the Einstein frame, using the simplifying assumptions made by the earlier authors.

The present evaluation of the density fluctuation differs from the earlier ones in two respects. One is the time at which the perturbation in the inflationary epoch is evaluated. We evaluate it exactly at the time when the characteristic wavelength starts growing bigger than the Hubble length. Then the mode functions are still oscillatory and physical quantities admit conventional interpretation. In the literature, however, the perturbation is actually evaluated when the wavelength has grown several times bigger than the the Hubble length and the mode functions have ceased to oscillate.

Although evaluated at two different times with qualitatively different mode functions, the density fluctuations turn out to have the same, nearly scale independent, spectum. It is interesting to note that our evaluation leaves the index $\nu$ of the Hankel function free, while that in the literature requires $\nu$ to be equal to $\frac{3}{2}$ to arrive at this spectrum. But the two evaluations do make a difference in the magnitude of the fluctuation, our result being greater by a factor of $2 \pi$.

The other point of difference with the earlier evaluation is the inclusion of the $\dot{\phi}$ term in $T_{00}$. In our way of evaluation, this term is as important as the $\phi$ term. These two sources of difference go to make our result larger by about a factor of 10 .

We hope to have made it clear that these sources of enhancement in our calculation over the earlier ones, although discussed here in the context of a specific model, is, in fact, quite general. But to arrive at the physically correct magnitude of $(\delta \rho / \rho)_{H}$, we need a better knowledge of the evolution of the density perturbation than what the approximate constancy of $\zeta$ suggests. Pending this investigation, a comparison of our result with the earlier ones indicates the magnitude of uncertainty in such a calculation.

One of us (SM) would like to thank Professor Paul J. Steinhardt for earlier discussions on related topics. He also gratefully acknowledges the hospitality at the Institute for theoretical Physics, University of Bern, which made these discussions possible.

\section{References}

[1] For a clear introduction to the inflationary scenario, see S.K. Blau and A.H. Guth in 300 years of Gravitation, edited by S.W. Hawking and W. Israel (Cambridge Univ. Press, 1987). 
[2] S.W. Hawking, Phys. Lett., 115B, 295 (1982).

[3] A.H. Guth and S.-Y. Pi, Phys. Rev. Lett. 49, 1110 (1982).

[4] J.M. Bardeen, P.J. Steinhardt and M.S. Turner, Phys. Rev. D28, 679 (1983). See also J.M. Bardeen, Phys. Rev. D22, 1882 (1980).

[5] For a summary of the inflationary models, see E.W. Kolb in Proc. of 42nd Scottish Univ. Summer School in Physics, Scotland, 1993.

[6] A.H. Guth, Phys. Rev. D23, 347 (1981)

[7] A.D. Linde, Phys. Lett., 108B, 389 (1982) ; A. Albrecht and P.J. Steinhardt, Phys. Rev. Lett. 48, 1220 (1982).

[8] A.D. Linde, Phys. Lett. 129B, 177 (1983)

[9] D. La and P.J. Steinhardt, Phys. Rev. Lett. 62, 376 (1969). See also C. Mathiazhagan and V.B. Johri, Class. Quan. Grav., 1, L29 (1984)

[10] P. Jordan, Z. Phys. 157, 112(1959).

[11] C. Brans and R.H. Dicke, Phys. Rev. 24, 924 (1961).

[12] A.M. Green and A.R. Liddle, Phys. Rev. D54, 2557 (1996).

[13] R.D. Reasenberg et al, Astrophys. J., 234, L219 (1979)

[14] T.S. Bunch and P.C.W. Davies, Proc. Roy. Soc., London, A360, 117 (1978) ; A.H. Guth and S.-Y. Pi, Phys. Rev. D32, 1899 (1985).

[15] E.W. Kolb and M.S. Turner, The early universe, (Addison-Wesley,1989)

[16] T. Padmanabhan, Structure formation in the universe (Cambridge Univ. Press, 1993)

[17] R. Holman, E.W. Kolb, S.L. Vadas, Y. Wang and E.J. Weinberg, Phys. Lett. 237, 37 (1990)

[18] E.W. Kolb, D.S. Salopek and M.S. Turner, Phys. Rev. D42, 3925 (1990)

[19] A.H. Guth and B. Jain, Phys. Rev. D45, 426 (1992)

[20] N.D. Birrell and P.C.W. Davies, Quantum fields in curved space ( Cambridge Univ. Press, 1982).

[21] It is well-known that positive and negative frequency mode functions cannot be defined if the momentum and mass terms in the mode equation cannot compensate for the expansion rate term. However here we need only consider those physical momenta whose wavelengths are not bigger than the Hubble length. The corresponding mode functions are oscillatory. 
[22] L.F. Abbott and M.B. Wise, Nucl. Phys., B244, 541 (1984).

[23] M. Abramowitz and I.A. Stegun, Handbook of mathematical functions (Dover,New York, 1972).

[24] The symbol for Hubble 'constant', $H(t)$, should cause no confusion with the one for the Hankel function, $H_{\nu}^{(1,2)}(z)$.

[25] This requirement in the Einstein frame is not as obvious as the corresponding one in the Jordan frame, where one requires the constancy of the $\psi$ from the onset of radiation domination. When converted to the Einstein frame it gives, of course, the same result for $t_{e}$ as in (4.4). 